\title{
HEMODYNAMIC STUDIES ON ESOPHAGEAL VARICES FORMATION IN THE PORTAL HYPERTENSIVE DOGS
}

\author{
HIDEAKI YAMANA, JINRYO TAKEDA, TERUO KAKEGAWA ${ }^{1)}$ \\ AND HIDEAKI TSUDA ${ }^{2)}$ \\ The First Department of Surgery ${ }^{1)}$ and Department of Anesthesiology ${ }^{21}$, \\ Kurume University School of Medicine, Kurume, 830, Japan
}

Received for publication July 30, 1980

\begin{abstract}
Many studies on the pathophysiology of the portal hypertension have been reported, but the studies on the contributory factors of the esophageal varices formation are few. Esophageal varices have been simply thought as a manifestation of the hepatofugal callaterals. Recently Inokuchi (1977) proposed a new hypothesis suggesting important role of hyperhemodynamic state due to an increased arteriovenous shunt in the gastric region. The purpose of this paper is to prove this new hypothesis in our newly developed portal hypertensive models. Angiographic and radioactive tracer studies were used for analysis of hemodynamic state in portal hypertensive dogs, and those studies showed clear evidences of an increased arteriovenous shunt as well as submucosal venovenous shunting in the upper gastric region.
\end{abstract}

\section{INTRODUCTION}

In the middle of 1930, Whipple and his colleagues introduced the concept that the portal hypertension was caused by outflow block of portal venous blood. Since then, many studies on the pathogenesis or pathophysiology of portal hypertension have been reported to prove this concept. Almost all studies suggested that the development of esophageal varices was a manifestation of defence mechanism building up numerous collaterals to reduce high portal venous pressure.

In 1977, Inokuchi et al proposed a new hypothesis (Inokuchi et al, 1977) suggesting a great role of hyperhemodynamic state in the gastric region due to an increased arteriovenous shunt rate to form the esophageal varices by their close clinical observations. This hypothesis is quite suggestive and interesting to clinicians who observe discrepancy between no esophageal varices with high portal presssure and notable esophageal varices with mild portal pressure. In addition of that discrepancy, it is also suggestive to researchers who have great difficulty to produce esophageal varices experimentally in animals.

Fortunately we were able to develop a new method of the entire liver compression (Yamana, 1980) to produce experimental portal hypertension with esophageal varices in the dog. We aimed in the present investigation to prove this new hypothesis on the formation of esophageal varices by hemodynamic studies of the stomach.

\section{MATERIAL AND METHOD}

Experiments were performed on 21 
mongrel dogs of either sex weighing between 11 and 19 kirograms. In eighteen dogs out of 21 , the entire liver compression was performed to produce portal hypertensive models. Animals were fasted for 18 hours before the operation and anesthetized with pentobarbital sodium, intubated orotracheolly and allowed to breath spontaneously. The femoral arterial pressure was monitored and a venous line was kept for continuous infusion of Ringer's solution during the operation. A catheter was cannulated into a branch of the superior mesenteric vein for measurement of portal venous pressure. Ligaments around the liver were divided carefully and the whole liver was wrapped and compressed with tensioning ligature of polypropylen
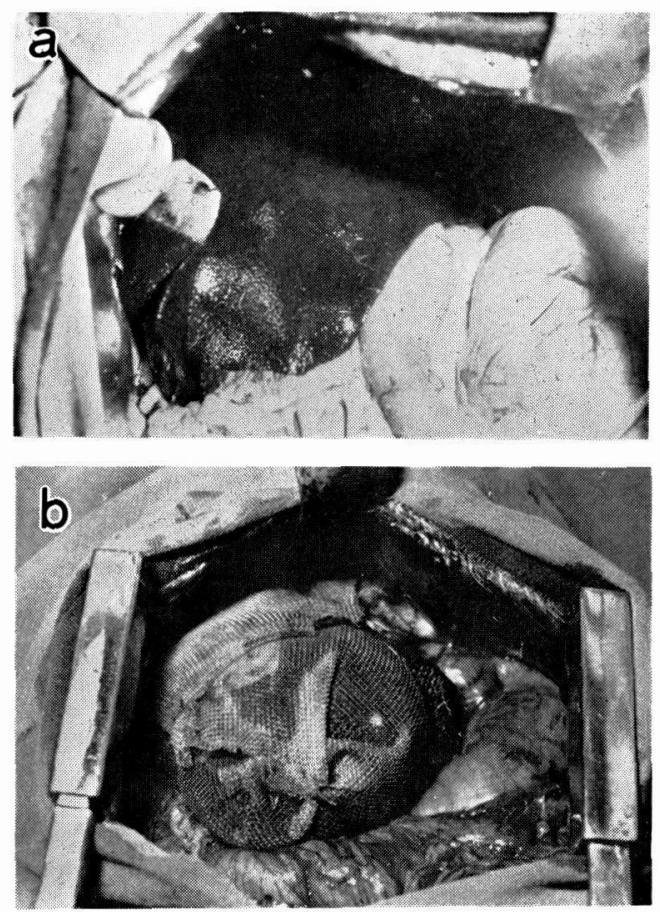

Fig. 1. Photographs of the liver before and after the liver compression with polypropylen mesh.

(a; before., b; after.) mesh (Davol/Usher's Marlex Mesh, DVL-1266). Special attension was paid to avoid constriction of extrahepatic veins and portal veins by the compression. Figure 1 shows photographs before and after the entire liver compression.

The first 15 dogs were sacrificed, and immediately the left gastric venography or arteriography was taken by injection of contrast medium for morphological observation of vessels in portal hypertension in 9 weeks after the operation. Microscopic studies were also performed on the stomach and esophagus.

The rest 3 portal hypertensive dogs were used for measurement of the gastric arteriovenous shunt rate by means of R. I. tracer ( ${ }^{131} \mathrm{I}-\mathrm{M}$. A. A.) technique with 3 normal $\operatorname{dog} s$ as a control. ${ }^{131}$ I-M. A. A. $(100 \mu \mathrm{ci})$ was injected slowly into the left gastric artery and animals were sacrificed in

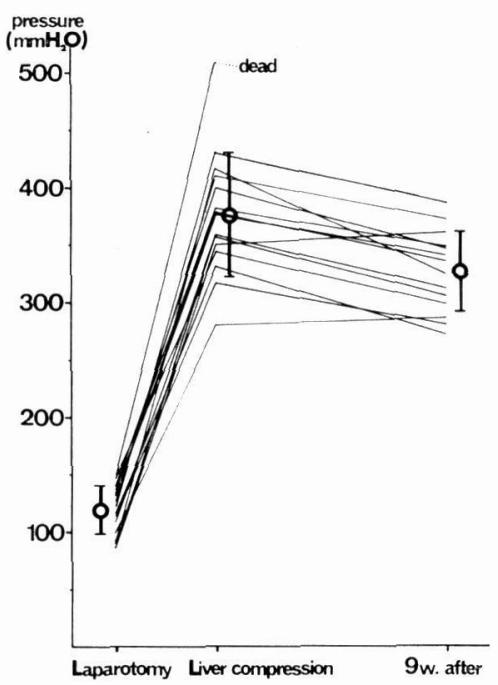

Fig. 2. The change in portal venous pressure before and after the entire liver compression in $15 \mathrm{dogs}$. 
3 minutes by $\mathrm{KCl}$ injection. R. I. counts per gram of each section of stomach (antrum and cardia), liver (internal left and right lobe), lung (inierior left and right lobe), spleen, and esophagus ( $3 \mathrm{~cm}$ upper portion of $\mathrm{E}-\mathrm{G}$ junction) were traced by Well type scintiration counter.

\section{RESULTS}

The changes in portal venous pressure before and after the liver compression in $15 \operatorname{dogs}$ were shown in Figure 2. The
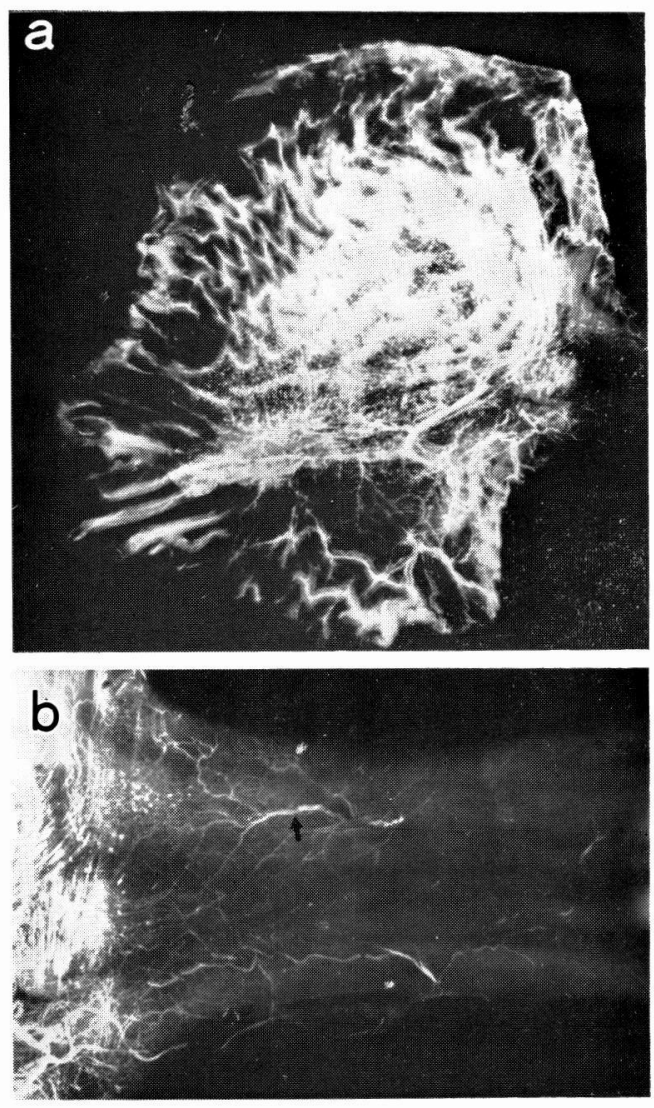

Fig. 3. Arteriographic findings of the stomach and esophagus.

(a) stomach., b; esopagus, arrow showes a submucosal vein.) average of initial portal venous pressure was $119 \pm 21 \mathrm{mmH}_{2} \mathrm{O}$ and raised up to $376 \pm 54 \mathrm{mmH}_{2} \mathrm{O}$ by the liver compression and dropped to $326 \pm 35 \mathrm{mmH}_{2} \mathrm{O}$ in 9 weeks after the operation.

The dogs used for R.I. study showed the following portal venous pressure: $121 \pm 17 \mathrm{mmH}_{2} \mathrm{O}$ in initial, $355 \pm 23 \mathrm{mmH}_{2} \mathrm{O}$ right after the liver compression, 346 $\pm 34 \mathrm{mmH}_{2} \mathrm{O}$ in 3 weeks after the operation.

Gastric arteriograms taken by hypaque injection into the left gastric artery showed marked hypervascularity in the mucosa of the upper gastric region (Figure 3-a). And an esophageal submucosal vein was clearly found on the esophageal portion of this arteriogram (Figure 3-b).

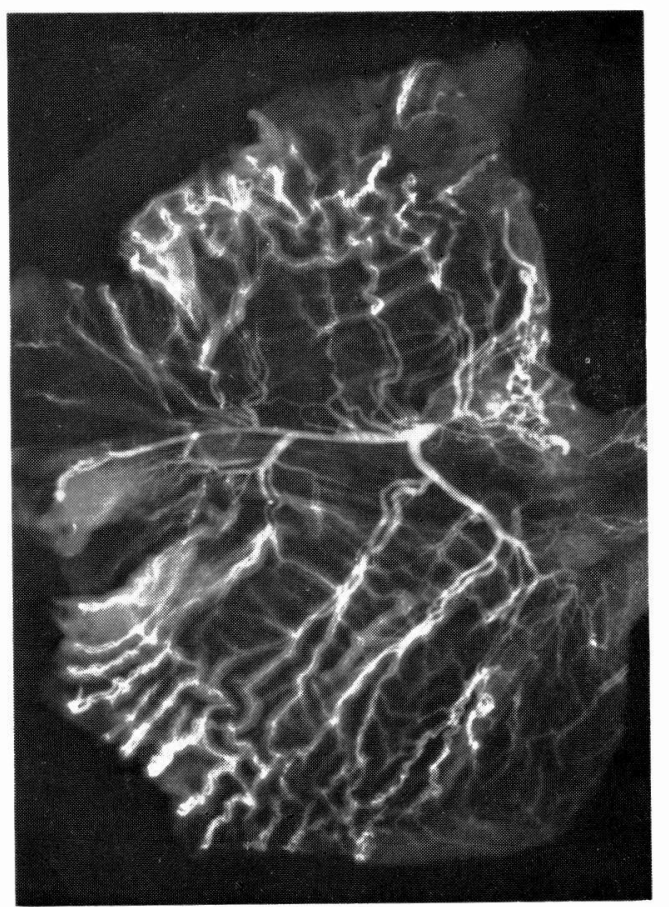

Fig. 4. Venografic finding of the stomach. 
Gastric venograms taken by hypaque injection into the left gastric vein showed a marked dilataion of gastric submucosal veins and development of venovenous shunting (Figure 4).

Photomicrographs taken after the hypaque injection in to the left gastric artery are shown in Figure 5. In the section of esophagus, a submucosal artery was filled with contrast medium and submucosal veins were also found to be filled with contrast medium (Figure 5-a). In the section of gastric lesser curvature, gastric submucosal arteries were filled with contrast medium and submucosal veins were also found to be filled with contrast medium (Figure 5-b).
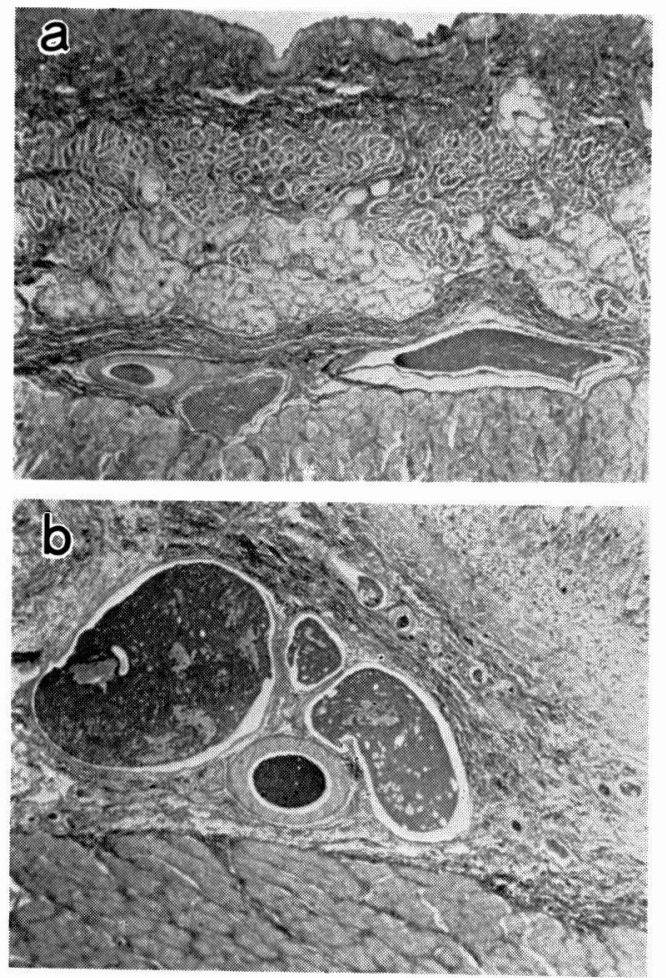

Fig. 5. Microscopic findings following the arteriography. ture.)
Photomicrographs taken after the hypaque injection into the left gastric vein are shown in Figure 6. In the section of esophagus, a marked dilatation of an esophageal submucosal vein filled with contrast medium was found (Figure 6-a). In the section of gastric lesser curvature, gastric submucosal veins showed a marked dilatation with contrast medium and submucosal arteris were also found to be filled with contrast medium (Figure 6-b).

Figure 7 showed the R. I. count rate of each section in 3 normal and 3 portal hypertensive dogs. In the normal dog, the average of R.I. count rate in the stomach was $98 \%$ (antrum, $25.5 \pm 5.1 \%$; cardia, $72.3 \pm 5.4 \%$ ), while the rest $2 \%$
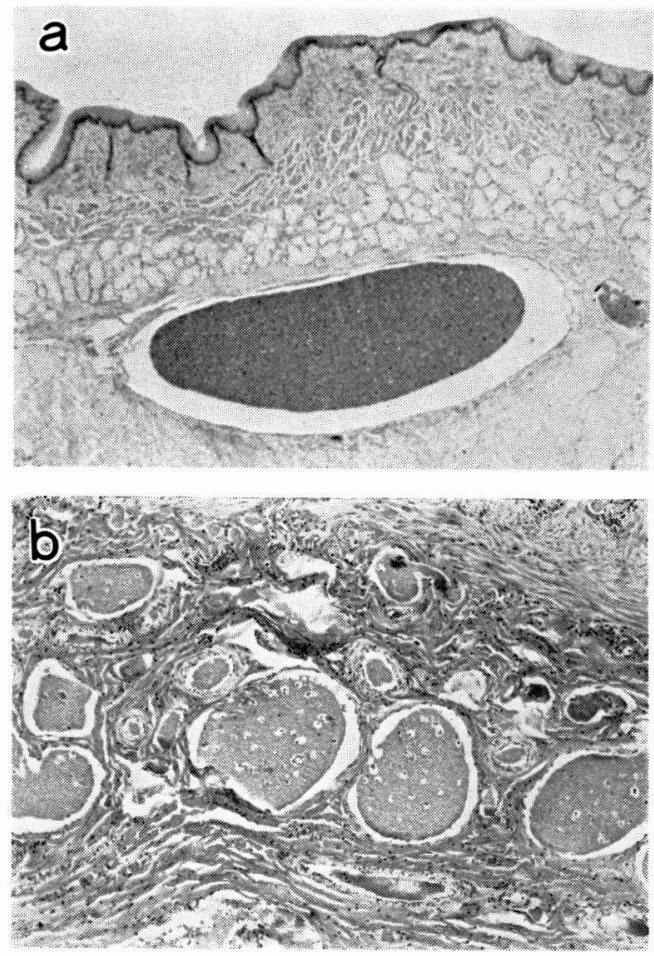

Fig. 6. Microscopic findings following the venography.

( a; esophagus., b; stomaoh of lesser curvature.) 


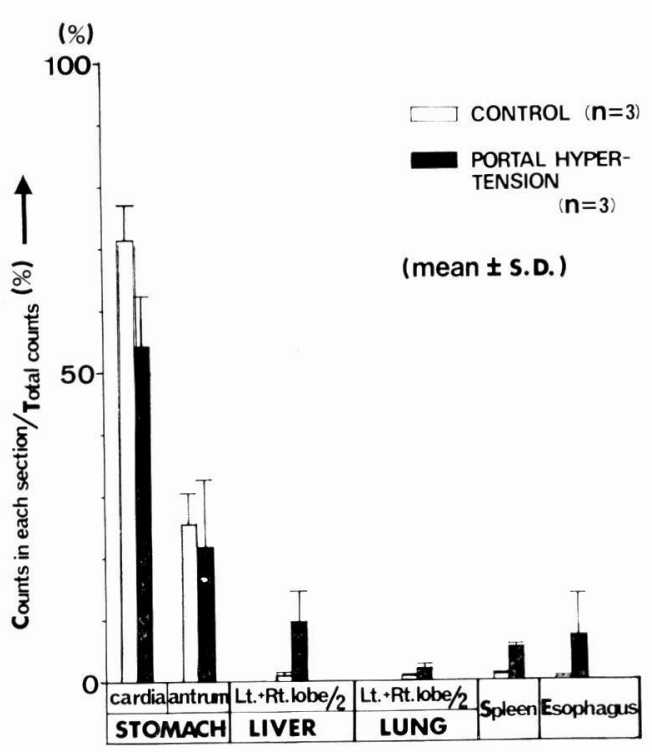

Fig. 7. Distribution of ${ }^{131} \mathrm{I}-\mathrm{MAA}$ in each section per gram.

of R. I. passed through arteriovenous shunts and was counted in other sections (liver, $0.8 \pm 0.2 \%$; lung, $0.7 \pm 0.1$ $\%$; spleen, $0.9 \pm 0.1 \%$; esophagus, $0.3 \pm$ $0.1 \%)$. The radioactivity of peripheral venous blood was not significantly above background. In the portal hypertensive dog, R. I. count rate in the stomach reduced to $76 \%$ (antrum, $21.8 \pm 9.7 \%$; cardia, $54.1 \pm 8.4 \%)$, so the rest $24 \%$ of R. I. passed through arteriovenous shunts and was counted in other sections (liver, $9.5 \pm 6.3 \%$; lung, $2.1 \pm 0.1$ $\%$; spleen, $5.6 \pm 0.2 \%$; esophagus, $7.2 \pm$ $5.2 \%)$.

\section{DISCUSSION}

Many studies on the gastric hemodynamics for explanation of gastric ulcer formation in liver cirrhosis with portal hypertension (Kato, 1979; Streicher, 1941; Palmer, 1957) have been reported. Manabe et al. (1977) reported that there was an increase in the gastric arteriovenous shunt rate in the greater curvature, but no increase in the lesser curvature in experimentally produced portal hypertension by simple portal venous constriction. On the other hand, there was an increased arteriovenous shunt rate in the lesser curvature in experimentally produced cirrhosis by $\mathrm{CCl}_{4}$ injection. Those data seemed to be indicating that the development of gastric arteriovenous shunt in the upper gastric region was not caused by simple blockade of portal venous outflow. Because of the great difficulty of producing esophageal varices experimentally, studies on mechanism of formation of esophageal varices have been based on clinical observations. Inokuchi and his colleagues (1977) proposed a new hypothesis proposing a great role of hyperhemodynamic state due to an increased arteriovenous shunt in the upper gastric region. This hypothesis, which was based on a close clinical observation, is in keeping with Tamiya and Thal's (1960) first success in experimental production of esophageal varices by making surgical splenic arteriovenous shunt with simultaneous splenic venous occlusion. This time we intended to prove this hypothesis in our newly developed portal hypertensive models which closely resemble the human pathology of the present subject (Takeda et al., 1980).

Angiographic findings suggested development of gastric arteriovenous shunting in portal hypertensive dogs compared to normotensive dogs. R. I. tracer study demonstrated $76 \%$ of radioactive macroaggregated albumin stayed in the stomach of portal hypertensive dogs, where $98 \%$ stayed in the normotensive dog's stomach which strongly suggested the rest $24 \%$ of radioactive substances passed through the arteriovenous shunt in portal hypertensive dogs. R.I. counts in the 
lung was, however, small, because of inconspicuous esophageal varices formation due to a short-lasting portal hypertension in the experimental model. If we use a longer-lasting portal hypertensive models, we may have obtained a greater shunt rate and a more radioactive count in the lung.

Our experimental data also suggested an important role of hyperhemodynamic state for esophageal varices formation. It can be speculated from our data that a hyperhemodynamic state in the upper gastric region and an outflow block of the gastric venous system associated with portal hypertension would contribute to esophageal varices formation, because congested blood in the upper gastric region has to flow away to a low pressure zone such as intrathoracic esophageal veins and resulting in esophageal venous dilatation. We presume that the increase in gastric arteriovenous shunt will be one of the important factors to form the esophageal varices.

\section{SUMMARY}

We intended to demonstrate an important role of hyperhemodynamic state on esophageal varices formation in our newly developed experimental portal hypertensive models. Our angiographic and R.I. tracer studies suggested that an increase in gastric arteriovenous shunt rate is one of the im- portant factors for esophageal varices formation.

\section{REFERENCES}

INOKUChi, K., Kobayashi, M., SAKu, M., NAGASUe, N., Iwaki, A. and Nakayama, S. (1977). Charactaristics of splanchnic portal circulation in portal hypertension as analysed by pressure study in clinical cases. Acta Hepatologica Japonica. 18, 891-898.

Kato, H. (1979). Gastric acid secretion in cases with portal hypertension. Jap. J. Gastroent. 76, 1427-1439.

Manabe, T., Suzuki, T. and Honjo, I. (1977). A hemodynamic study on the development of gastric mucosal injury in liver 1 cirrhosis. Jap. J. Gastroent. 74, 40-51.

Palmer, E. D. (1957). Erosive gastritis in cirrhosis. Influence of portal hypertension on the gastric mucosa. Am. J. Dig. Dis. 2, 31-38.

Streicher, M. H. (1941). Effect of hepatic damage on gastric acidity. Arch. Surg. 43, 74-81.

Tamiya, T. and Thal, A. P. (1960). Esophageal varices produced experimentally in the dog. Surg. Gynecol. Obstet. 111, 147-154.

TaKeda, J., Yamana, H., FuKushima, H., KaTSUKI, G., EDAKUNI, S., TAKEOKA, A. and UMETANI, T. (1980). Experimental production of portal hypertension and esophageal varices in dogs by the entire liver compression method. Jap. J. Gastroent. Surg. 13. 1008-1015.

YAMANA, H. (1980). Experimental production of portal hypertension by an entire liver compression in dogs. Jpn. J. Cli. Exp. Med. 57, 1867-1870. 